\title{
Photodissociation in proto-planetary nebulae
}

\section{Hydrodynamical simulations and solutions for low-velocity multi-lobes}

\author{
G. García-Segura
}

Instituto de Astronomía-UNAM, Apdo Postal 106, Ensenada 22800, Baja California, Mexico

e-mail: ggs@astrosen.unam.mx

Received 13 August 2010 / Accepted 9 September 2010

\begin{abstract}
Aims. We explore the effects of photodissociation at the stages of post-asymptotic giant branch stars to find a mechanism able to produce multi-polar shapes.

Methods. We perform two-dimensional gasdynamical simulations to model the effects of photodissociation in proto-planetary nebulae.

Results. We find that post-asymptotic giant branch stars with $\sim 7000 \mathrm{~K}$ or hotter are able to photodissociate a large amount of the circumstellar gas. We compute several solutions for nebulae with low-velocity multi-lobes. We find that the early expansion of a dissociation front is crucial to understand the number of lobes in proto-planetary nebulae.

Conclusions. A dynamical instability appears when cooling is included in the swept-up molecular shell. This instability is similar to the one found in photoionization fronts, and it is associated with the thin-shell Vishniac instability. The dissociation front exacerbates the growth of the thin-shell instability, creating a fast fragmentation in shells expanding into media with power-law density distributions such as $r^{-2}$.
\end{abstract}

Key words. hydrodynamics - stars: AGB and post-AGB - planetary nebulae: general

\section{Introduction}

Several surveys of proto-planetary nebulae (PPNs) and planetary nebulae (PNs) (Sahai et al. 2007a; Chu et al. 1987; Balick 1987; Schwarz et al. 1992; Stanghellini et al. 1993; Manchado et al. 1996a; Manchado et al. 1996b) show a rich variety of shapes, and they have been cataloged in a series of morphological classes: bipolar, elliptical, point-symmetric, irregular, spherical, quadrupolar, and multi-polar. While the first five former classes have been reproduced with different scenarios by hydrodynamical and magnetohydrodynamical simulations (Icke 1988, 1989; Soker \& Livio 1989; Mellema et al. 1991; Icke et al. 1992; Frank \& Mellema 1994; Dwarkadas et al. 1996; Różyczka \& Franco 1996; García-Segura 1997; García-Segura et al. 1999; Rijkhorst et al. 2005; García-Segura et al. 2005), the last two former classes have eluded any attempt of modeling, except the one by Rijkhorst et al. (2005) and Matt et al. (2006). In general, all previous scenarios are based on the collimation of winds. Two examples of low-expanding, multi-polar PPNs are IRAS 19024+0044 (Sahai et al. 2007b) and IRAS 19475+3119 (Sahai et al. 2007c). It is interesting that these two nebulae neither show any kinematic fingerprint in their optical spectra nor a perfect symmetry.

Only recently, the hypothesis that bipolar and multipolar lobes of PNs are the result of ionization and illumination was proposed by Kwok (2010). He suggested that multipolar nebulae with similar lobe sizes are not caused by simultaneous ejection of matter in several directions, but by the leakage of UV photons into those directions. Therefore, the explanation to the multipolar phenomenon does not lie in the dynamical ejection, but in how such holes were formed. The similarity in sizes of the multiple lobes was therefore naturally explained, and no simultaneous ejection was required.

According to Kwok's idea, it is necessary to find a mechanism that is able to create holes in the surrounding medium of PPNs, different to stellar winds or collimated outflows of any kind, such as jets originated by accretion disks (Livio \& Pringle 1997), or the effects from photoionization (García-Segura \& Franco 1996), because post-asymptotic giant branch stars (postAGBs) are still not hot enough to produce a considerable ionization of the medium.

Diaz-Miller et al. (1998) made an important contribution to the study of photodissociation regions (PDRs) around mainsequence stars. They outlined the importance of PDRs for lowtemperature stars (down to $7500 \mathrm{~K}$ ), because the number of non-ionizing photons in the range of $912 \AA<\lambda<1100 \AA$ is several orders of magnitude larger than the ionizing photons $(\lambda<912 \AA)$, which are able to dissociate the outlying molecular gas, forming PDRs.

A number of previous studies (Latter et al. 2000, and references therein) have outlined the importance of PDRs in the context of PNs. However, previous models (Natta \& Hollenbach 1998) have focused on stellar temperatures above $30000 \mathrm{~K}$, or even higher, such as the case of the Helix Nebula (Henney et al. 2007).

Inspired by Kwok's idea, we explore the effects of photodissociation at the stages of post-AGBs, when stars still have low temperatures $(\sim 7000 \mathrm{~K})$, i.e., they are still in the socalled transition time $(5000 \leq 10000 \mathrm{~K})$, and it is found that 
PDRs are able to explain several observational features found in PPNs. The transition time of post-AGBs is not yet fully understood (Stanghellini \& Renzini 2000), since the mass-lost rate at these stages is basically unknown. Stellar evolution calculations of PN central stars usually avoid this stage and start at $10000 \mathrm{~K}$ (Vassiliadis \& Wood 1994), although there is a model for $3_{\text {ZAMS }} M_{\odot}$ that starts at $\sim 6000 \mathrm{~K}$ (Blöcker \& Schönberner 1990). In the context of the present paper, long transition times $\geq 10000 \mathrm{yr}$ (low mass stars) will produce dynamically, developed PDRs, while transition times shorter than $1000 \mathrm{yr}$ (massive stars) do not give enough time to develop a structured PDR and will form rapidly photoionized nebulae.

In this paper, we use the study by Diaz-Miller et al. (1998) as a starting point, and merely replace the constant density condition by a numerical integration for any density distribution. The paper is structured as follows: Sect. 2 describes the numerical models and physical approaches used in this study. Section 3 presents two-dimensional results of PPNs. Section 4 discusses the results and gives a summary.

\section{Numerical models and physical approaches}

The simulations have been performed with the magnetohydrodynamical code ZEUS-3D version 3.4 (Clarke 1996), developed by M. L. Norman and the Laboratory for Computational Astrophysics. This is a finite-difference, fully explicit Eulerian code descended from the code described in Stone \& Norman (1992). ZEUS-3D does not include radiation transfer, but we have implemented a simple approximation (Diaz-Miller et al. 1998) to derive the location of the dissociation front for arbitrary density distributions, similar to the approximation used to locate the ionization front (Tenorio-Tagle 1979; Bodenheimer et al. 1979; Franco et al. 1989; García-Segura \& Franco 1996). This is done by assuming that dissociation equilibrium holds at all times, and that the gas is fully dissociated inside the PDR. One can define the PDR equilibrium radius similarly to the Strömgren radius (Diaz-Miller et al. 1998), and the position of the dissociation front in any given direction $(\theta, \phi)$ from the photodissociating source is given by

$4 \pi \int_{R_{\mathrm{HII}}}^{R_{\mathrm{PDR}}} n_{\mathrm{H}^{0}} n_{\mathrm{tot}} \alpha_{f} r^{2} \mathrm{~d} r=\langle p\rangle S_{\mathrm{D}}$,

where $n_{\mathrm{H}^{0}}$ is the density of neutral hydrogen, $n_{\mathrm{tot}}=n_{\mathrm{H}^{0}}+2 n_{\mathrm{H}_{2}}$ is the total proton density, $\alpha_{f}=1.37 \times 10^{-17}$ (Hollenbach \& Salpeter 1971), $\langle p\rangle=0.15$ (Abgrall et al. 1992; Draine \& Bertoldi 1996), and $S_{\mathrm{D}}$ the rate of dissociating photons. In the context of this paper, we assume that $R_{\mathrm{HII}}=0$. The left side of Eq. (1) is the number of $\mathrm{H}_{2}$ molecules formed in a given volume, while the right side is the total number of photodissociations per unit time, where $\langle p\rangle$ is the average dissociation probability for the wavelength range 912-1100 .

The stellar parameters are taken from a model for $M_{\text {ZAMS }}=$ $2.5 M_{\odot}$ (Vassiliadis \& Wood 1994), and using a black-body approximation similar to Villaver et al. (2002) for $T_{\text {eff }}=$ $6-7-8-10 \times 10^{3} \mathrm{~K}$, we obtain $\log \left(S_{\mathrm{D}}\right) \sim 42,43,44,45 \mathrm{~s}^{-1}$.

The models include the cooling curves by Dalgarno \& McCray (1972) and MacDonald \& Bailey (1981). The cooling cutoff temperature of the molecular gas is set to $10 \mathrm{~K}$. Finally, the photodissociated gas is always kept at $10^{3} \mathrm{~K}$, so no cooling curve is applied to the PDRs (unless there is a shock inside the PDR).

Equatorial density enhancements are introduced in the twodimensional models using Eqs. (2) to (5) in García-Segura et al. (1999), based on the wind-compression equations by
Bjorkman \& Cassinelli (1993). Here , $\Omega=v_{\text {rot }} / v_{\text {crit }}$ gives the proximity of the star to critical rotation (see Eq. (6) in GarcíaSegura et al. 1999). We assume that critical rotation (or close) is achieved at some point in the blueward excursion (Langer 1998; Heger \& Langer 1998) during the transition time, similar to the model computed for $12 M_{\odot}$ (Chita et al. 2008). Common envelope scenarios probably produce similar effects, although there are not yet any available equations in the literature.

\section{Two-dimensional results}

The first two-dimensional model consists of three stages. In the initial stage, we initialized the computational domain with a spherical AGB wind with $\dot{M}=1 \times 10^{-5} M_{\odot} \mathrm{yr}^{-1}$ and $v_{\infty}=10 \mathrm{~km} \mathrm{~s}^{-1}$. Our grid consists of $200 \times 180$ equidistant zones in $r$ and $\theta$, respectively. The innermost radial zone lies at $r_{\mathrm{i}}=2.725 \times 10^{-3} \mathrm{pc}$, and the outermost zone at $r_{\mathrm{o}}=0.109 \mathrm{pc}$. The angular extent is $90^{\circ}$.

In the second stage, we introduce a second wind at the inner boundary with the same parameters, but it is assumed that the star is near critical rotation with $\Omega=0.98$. This is done for a period of $10^{4} \mathrm{yr}$. We do not include here a smooth transition (from $\Omega=0 \longrightarrow 0.98$ ) for simplicity. These two stages are then used as initial conditions for a third stage. We set all velocities in the grid to zero. This is done to avoid the expansion of the AGB wind out of the grid and to compute only the expansion of the PDR.

The first snapshot in Fig. 1 (at $1000 \mathrm{yr}$ ) shows the structure generated by both AGB winds, an outer spherical wind almost at the edge of the grid, followed by a bipolar wind. This bipolarity is owing to the smaller values of the radial velocity toward the equator in the AGB wind with critical rotation (see Eq. (2) in García-Segura et al. 1999), which produces an apparent empty space because of a rarefaction wave in the wind (dark areas in the plot). The dark areas would be filled with gas using a transition, evolving wind with $\Omega=0 \longrightarrow 0.98$.

In the third stage, we simulate the conditions of a post-AGB wind near critical rotation $(\Omega=0.98)$, decreasing the mass-loss rate down to $\dot{M}=1.73 \times 10^{-7} M_{\odot} \mathrm{yr}^{-1}$. Here, we assume that the star has reached $7000 \mathrm{~K}$, and the corresponding UV photons reach $S_{\mathrm{D}}=10^{43} \mathrm{~s}^{-1}$. As before, we artificially set the wind velocity to zero, just to compute only the contribution from the thermal expansion of the photodissociated gas. By doing this, we are also assuming at the same time that the post-AGB star is not able to drive an efficient wind, because the stellar temperature is not high enough to produce a line-driven wind.

Figure 1 shows the evolution of the gas density during 8000 yr. During the first thousand years, the lower density at the polar region allows the PDR to expand in the form of beams, digging low-density tunnels, preferentially at the polar axis (first snapshot in Fig. 1). The gas is evacuated and pushed sideways in those beams because of the sudden increase of the temperature (from 10 to $1000 \mathrm{~K}$ ) in the PDR. During the second thousand years (second snapshot in Fig. 1), the growth of the beam at the polar axis ends because of the lateral expansion of the two adjacent beams, blocking the UV field owing to the increase of density at the axis. At the end of this phase at $2000 \mathrm{yr}$, two thin lobes (0.08 pc long) are formed at each hemisphere (second snapshot in Fig. 1), producing a quadrupolar shape.

Meanwhile, the density gradient between pole and equator in the PDR close to the central star produces a flow directed toward the polar axis. This bipolar flow converges at high latitudes, and is able to increase the density up to the point of producing a self-blocking of the UV field between 2000 and $3000 \mathrm{yr}$. At this 
G. García-Segura: Photodissociation in proto-planetary nebulae
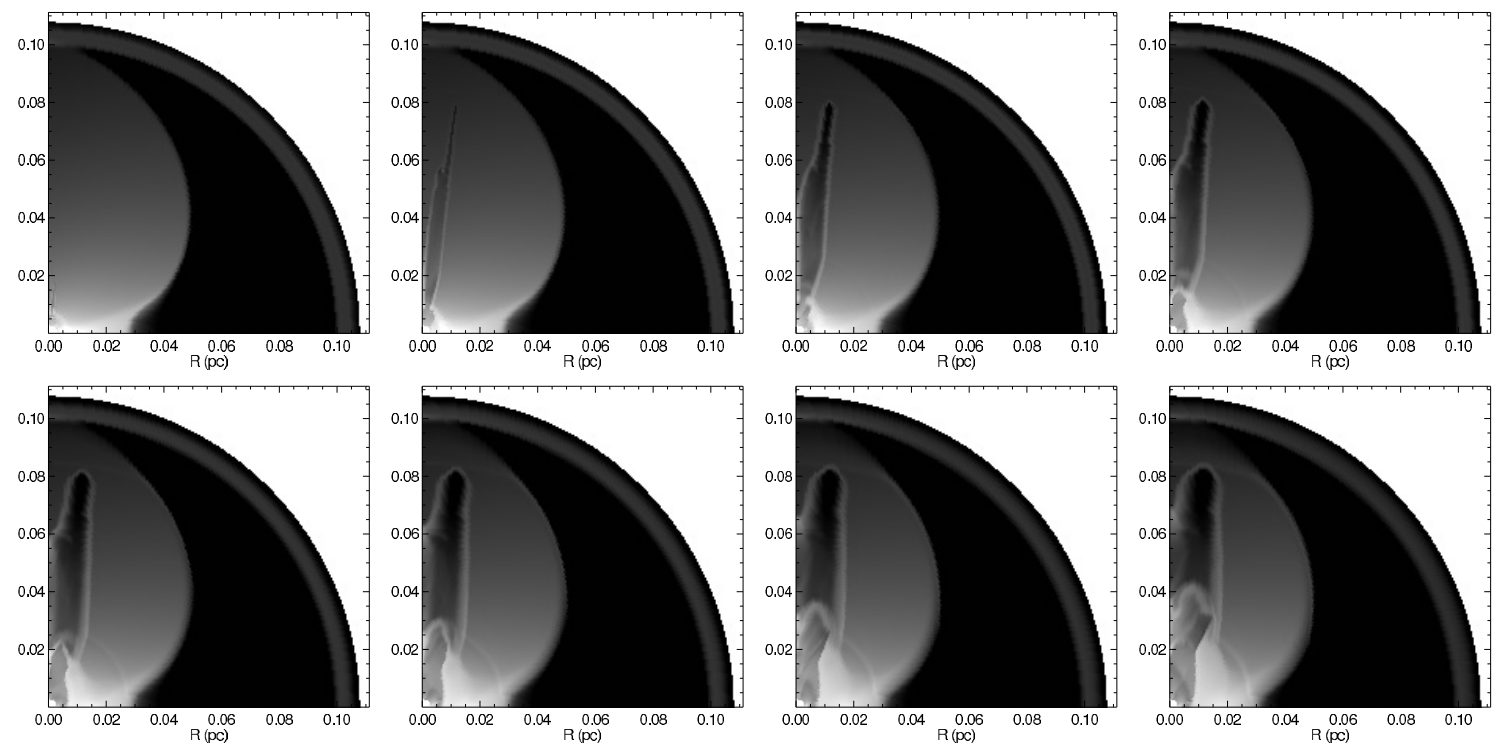

Fig. 1. Snapshots of gas density at 1, 2, 3, 4 (top), and 5, 6, 7, $8 \times 10^{3} \mathrm{yr}($ bottom $)(\log (\min )=-22, \log (\max )=-18)$.

Table 1. Sample of models.

\begin{tabular}{lcc}
\hline \hline model & $\dot{M}_{\mathrm{AGB}}\left(M_{\odot} \mathrm{yr}^{-1}\right)$ & $\Omega$ \\
\hline $80-\mathrm{a}$ & $0.60 \times 10^{-5}$ & 0.80 \\
$80-\mathrm{b}$ & $0.50 \times 10^{-5}$ & 0.80 \\
$50-\mathrm{a}$ & $0.40 \times 10^{-5}$ & 0.50 \\
$50-\mathrm{b}$ & $0.38 \times 10^{-5}$ & 0.50 \\
\hline
\end{tabular}

moment, the PDR contracts and becomes confined by this flow at $\sim 0.01 \mathrm{pc}$, and the external lobes cool down to form hydrogen molecules. After $3000 \mathrm{yr}$, the PDR again expands in the polar direction in a bipolar or quadrupolar form (the dissociation front has funnel shape).

The last snapshot in Fig. 1 (at 8000 yr) shows the final development of our model. At $0.02 \mathrm{pc}$, a high-density blob produced by the convergence of two oblique shocks defines the position of the dissociation front at the polar axis. Above this blob, the remnant lobes collimate the expansion of the molecular flow beyond the dissociation front. At $0.045 \mathrm{pc}$, the molecular outflow reaches values of $8.7 \mathrm{~km} \mathrm{~s}^{-1}$.

The initial expansion of a dissociation front could be very sensitive to the conditions found at the density ramp. To illustrate this behavior, we have computed a sample of four models in which we have changed the equator to pole density ratio by changing the stellar rotation $(\Omega=0.80,0.50)$, with a slightly different mass-loss rate for those with the same rotation (Table 1). The other computational inputs have the same properties as the former model, except that we skip the first stage (the initial spherical wind), and directly initialize the computational mesh with the wind near critical rotation. The post-AGB wind is not included either, only the UV field. The results are shown in Fig. 2, with snapshots at 9000 yr. The angular extent is now $180^{\circ}$ in $\theta$, with 360 zones. We have introduced a $1 \%$ random noise in the initial density to allow for differences between the northern and the southern hemispheres, to emphasize that the lobes could reach different sizes and be formed at different angles, i.e., they are not necessarily symmetric. The expansion velocity of the lobes in Fig. 2 is lower than $1 \mathrm{~km} \mathrm{~s}^{-1}$ with respect to the AGB wind ( $=0$ in the simulations).
We cannot discuss each model in detail here, but give a short summary. We find that the early expansion of a dissociation front is crucial to understand the number of lobes in the models, and we also find that the number of lobes is inversely proportional to the equator/pole density ratio. A PDR is able to expand in more directions when the ratio is low. For example, in the extreme case of Fig. 1, the PDR only occupies a few cells in the polar region, and the dissociation front digs a tunnel only through the polar axis (small solid angle), while in the case of model 50-b (Fig. 2), the initial PDR occupies many cells down to much lower latitudes, and the PDR expands into a larger solid angle. In the ideal case of a spherical wind (not shown in the figure), the twodimensional result has the shape of a daisy flower, because the PDR expands in all directions. Note that the development of instabilities in a dissociation front is crucial to understand the expansion of PDRs (see Sect. 4).

\section{Discussion and summary}

García-Segura \& Franco (1996) noted that a D-type dissociation front preceded by a radiative shock should be unstable, similarly to the ionization-shock (I-S) front instability of a D-type ionization front. By analogy to the I-S front instability (Giuliani 1979), we called it here dissociation-shock (D-S) front instability. The dynamical behavior of the D-S front instability is equivalent to the one of the I-S front instability (García-Segura \& Franco 1996; Whalen \& Norman 2008), the only difference is that the temperature of a PDR is around one order of magnitude lower than that of a HII region. This causes a lower pressure gradient between the molecular and the dissociated gas, and consequently, the growth, development, and dynamics of the D-S front instability would be less violent than that of the I-S front instability.

At the onset of the D-S front instability, the CSM gas is forced to cross oblique shocks, and the gas acquires a transverse component in velocity (Vishniac 1983; Mac Low \& Norman 1993). Thus, the gas piles up in the "valleys" (by analogy), enhancing the column density, but the gas is cleared out from the "peaks", therefore lowering the column density. The radiation field notices the redistribution of the gas and a number of photons can now reach deeper regions in the directions where the 

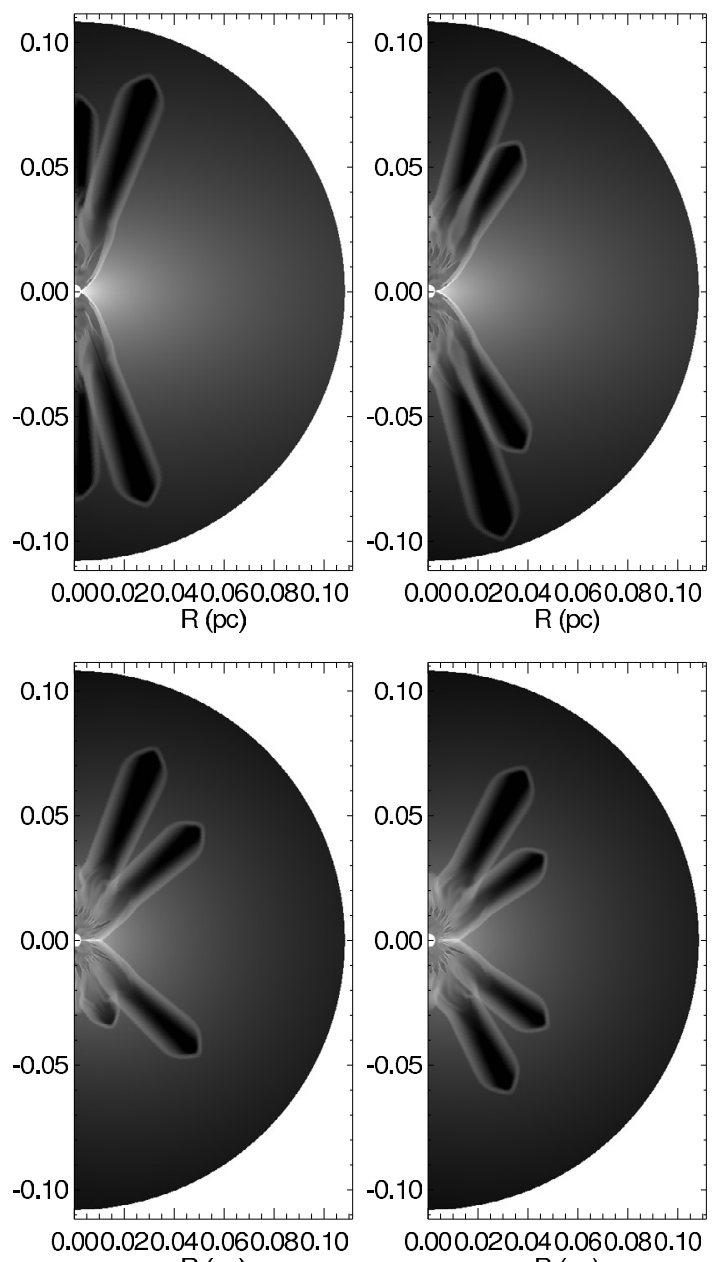

$\mathrm{R}(\mathrm{pc})$

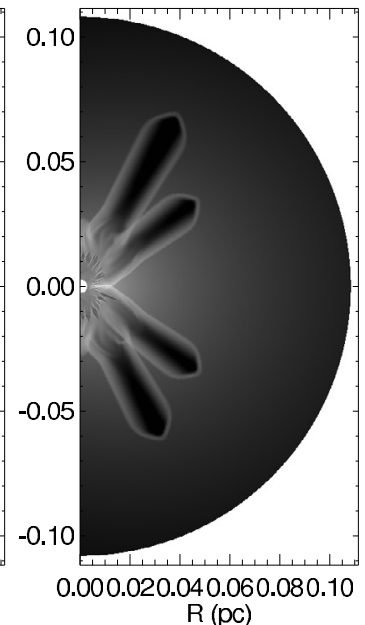

$\mathrm{R}(\mathrm{pc})$

Fig. 2. From left to right, top to bottom: gas density of models 80-a, $80-b, 50-a, 50-b$ at 9000 yr of the evolution $(\log (\min )=-22$, $\log (\max )=-18)$. Model numbers relate the approach to critical rotation (in percent).

"peaks" are located. Because the inertia of the swept-up shell decreases at the "peaks", the dissociated gas expands and accelerates the leading shock, steepening the angle of the oblique shocks. If the CSM has a decreasing slope in density (such as $r^{-2}$ in AGB winds), the growth of this instability becomes catastrophic. Dissociation fronts should be also subject to a shadowing instability similar to ionization fronts (Williams 1999; Whalen \& Norman 2008), which probably triggers the development of the D-S front instability.

We have shown here that photodissociation produces an important fragmentation in PPNs via the D-S front instability, and is able to shape complicated structures, like quadrupolar and multipolar lobes. Then, according to Kwok's idea, photodissociation is the mechanism able to create holes in the surrounding medium of post-AGBs. Once the holes are formed, the postAGB wind could be channeled through them, forming multipolar bubbles.

In a future article, we will perform calculations using new evolutionary tracks for post-AGBs, including other inputs such

as stellar winds, with resolution studies for the D-S front instability, and with 3-D solutions.

Acknowledgements. G.G.-S. thanks the referee, Vincent Icke, for his valuable comments which improved the article, and thanks Michael L. Norman and the Laboratory for Computational Astrophysics (LCA) for the use of ZEUS-3D. This work has been partially supported by the grant DGAPAUNAM IN100410-3.

\section{References}

Abgrall, H., Le Bourlot, J., Pineau des Forets, G., Roueff, E., \& Heck, L. 1992, A\&A, 253, 525

Balick, B. 1987, AJ, 94, 671

Bjorkman, J. E., \& Cassinelli, J. P. 1993, ApJ, 409, 429

Blöcker, T., \& Schönberner, D. 1990, A\&A, 240, L11

Bodenheimer, P., Tenorio-Tagle, G., \& Yorke, H. W. 1979, ApJ, 233, 85

Chita, S. M., Langer, N., van Marle, A. J., García-Segura, G., \& Heger, A. 2008, A\&A, 488, L37

Chu, Y.-H., Jacoby, G. H., \& Arendt, R. 1987, ApJS, 64, 529

Clarke, D. A. 1996, ApJ, 457, 291

Dalgarno, A., \& McCray, R. A. 1972, ARA\&A, 10, 375

Diaz-Miller, R. I., Franco, J., \& Shore, S. N. 1998, ApJ, 501, 192

Draine, B. T., \& Bertoldi, F. 1996, ApJ, 468, 269

Dwarkadas, V. V., Chevalier, R. A., \& Blondin, J. M. 1996, ApJ, 457, 773

Franco, J., Tenorio-Tagle, G., \& Bodenheimer, P. 1989, Rev. Mexicana Astron. Astrofis., 18, 65

Frank, A., \& Mellema, G. 1994, A\&A, 289, 937

García-Segura, G. 1997, ApJ, 489, L189

García-Segura, G., \& Franco, J. 1996, ApJ, 469, 171

García-Segura, G., Langer, N., Różyczka, M., \& Franco, J. 1999, ApJ, 517, 767

García-Segura, G., López, J. A., \& Franco, J. 2005, ApJ, 618, 919

Giuliani, J. L. 1979, ApJ, 233, 280

Heger, A., \& Langer, N. 1998, A\&A, 334, 210

Henney, W. J., Williams, R. J. R., Ferland, G. J., Shaw, G., \& O’Dell, C. R. 2007, ApJ, 671, L137

Hollenbach, D. J., \& Salpeter, E. E. 1971, ApJ, 163, 155

Icke, V. 1988, A\&A, 202, 177

Icke, V., Preston, H. L., \& Balick, B. 1989, AJ, 97, 462

Icke, V., Balick, B., \& Frank, A. 1992, A\&A, 253, 224

Kwok, S. 2010, PASA, 27, 174

Langer, N. 1998, A\&A, 329, 551

Latter, W. B., Dayal, A., Bieging, J. H., et al. 2000, ApJ, 539, 783

Livio, M., \& Pringle, J. E. 1997, ApJ, 468, 835

MacDonald, J., \& Bailey, M. E. 1981, MNRAS, 197, 995

Mac Low, M.-M., \& Norman, M. L. 1993, ApJ, 407, 207

Manchado, A., Guerrero, M., Stanghellini, L., \& Serra-Ricart, M. 1996a, The

IAC Morphological Catalog of Northern Galactic Planetary Nebulae, ed. Instituto de Astrofísica de Canarias

Manchado, A., Stanghellini, L., \& Guerrero, M. 1996b, ApJ, 466, L95

Matt, S., Frank, A., \& Blackman, E. G. 2006, ApJ, 647, L45

Mellema, G., Eulderink, F., \& Icke, V. 1991, A\&A, 252, 718

Natta, A., \& Hollenbach, D. 1998, A\&A, 337, 517

Rijkhorst, E.-J., Mellema, G., \& Icke, V. 2005, A\&A, 444, 849

Różyczka, M., \& Franco, J. 1996, ApJ, 469, L127

Sahai, R., Morris, M., Sánchez-Contreras, C., \& Claussen, M. 2007a, AJ, 134, 2200

Sahai, R., Sánchez-Contreras, C., \& Morris, M. 2007b, ApJ, 620, 948

Sahai, R., Sánchez-Contreras, C., Morris, M., \& Claussen, M. 2007c, ApJ, 658, 410

Schwarz, H. E., Corradi, R. L. M., \& Melnick, J. 1992, A\&AS, 96, 23

Soker, N., \& Livio, M. 1989, ApJ, 339, 268

Stanghellini, L. , \& Renzini, A. 2000, ApJ, 542, 308

Stanghellini, L., Corradi, R. L. M., \& Schwarz, H. E. 1993, A\&A, 276, 463

Stone, J. M., \& Norman, M. L. 1992, ApJS, 80, 753

Tenorio-Tagle, G. 1979, A\&A, 71, 59

Vassiliadis, E., \& Wood, P. 1994, ApJS, 92, 125

Villaver, E., Manchado, A., \& García-Segura, G. 2002, ApJ, 581, 1204

Vishniac, E. T. 1983, ApJ, 274, 152

Whalen, D. J., \& Norman, M. L. 2008, ApJ, 672, 287

Williams, R. J. R. 1999, MNRAS, 310, 789 